\title{
Towards the operational estimation of a radiological plume using data assimilation after a radiological accidental atmospheric release
}

\author{
Victor Winiarek $^{\mathrm{a}}$, Julius Vira ${ }^{\mathrm{b}}$, Marc Bocquet ${ }^{\mathrm{a}, \mathrm{c}}$, Mikhail Sofiev ${ }^{\mathrm{b}}$, Olivier Saunier ${ }^{\mathrm{d}, \mathrm{a}}$ \\ ${ }^{a}$ Université Paris-Est, CEREA, joint laboratory École des Ponts ParisTech and EDF RED, Champs-sur-Marne, France \\ ${ }^{b}$ Finnish Meteorological Institute, Helsinki, Finland \\ ${ }^{c}$ INRIA, Paris Rocquencourt research centre, France \\ ${ }^{d}$ Institute of Radiation Protection and Nuclear Safety, BP 17, 92262, Fontenay-aux-Roses, France
}

\begin{abstract}
In the event of an accidental atmospheric release of radionuclides from a nuclear power plant, accurate real-time forecasting of the activity concentrations of radionuclides is required by the decision makers for the preparation of adequate countermeasures. The accuracy of the forecast plume is highly dependent on the source term estimation. On several academic test cases, including real data, inverse modelling and data assimilation techniques were proven to help in the assessment of the source term.

In this paper, a semi-automatic method is proposed for the sequential reconstruction of the plume, by implementing a sequential data assimilation algorithm based on inverse modelling, with a care to develop realistic methods for operational risk agencies. The performance of the assimilation scheme has been assessed through the intercomparison between French and Finnish frameworks. Two dispersion models have been used: PolaIR3D and Silam developed in two different research centres. Different release locations, as well as different meteorological situations are tested. The existing and newly planned surveillance networks are used and realistically large multiplicative observational errors are assumed. The inverse modelling scheme accounts for strong error bias encountered with such errors. The efficiency of the data assimilation system is tested via statistical indicators. For France and Finland, the average performance of the data assimilation system is strong. However there are outlying situations where the inversion fails because of a too poor observability. In addition, in the case where the power plant responsible for the accidental release is not known, robust statistical tools are developed and tested to discriminate candidate release sites.
\end{abstract}

This article has been published in Atmospheric Environment with the reference:

Winiarek, V., Vira, J., Bocquet, M., Sofiev, M., Saunier, O., 2011. Towards the operational estimation of a radiological plume using data assimilation after a radiological accidental atmospheric release. Atmos. Env. 45. 2944-2955.

Keywords: Data assimilation, Atmospheric dispersion, Radionuclides, Emergency response

\section{Introduction}

\subsection{Context}

Emergency centres that are in charge of real-time estimation of the impact of an accidental release of pollutant, are getting interested in testing and implementing data assimilation techniques for source term estimation as well as for plume dispersion forecast.

In the case of the IRSN, the French nuclear safety agency, a simplistic Gaussian plume model was used so

Email address: victor.winiarek@cerea.enpc.fr (Victor Winiarek)

Preprint submitted to Atmospheric Environment far as an operational forecast model. An Eulerian modelling platform, $l d X$ based on the PoLYPHEMus platform (Quélo et al., 2007), is being implemented and should serve as their reference forecast tool.

Since the beginning of this century, academic researchers have been developing and testing, sometimes with real data, inverse modelling techniques for accidental release of pollutant, using Gaussian, Eulerian and Lagrangian models. With increasing confidence, they were able to demonstrate, at least at an academic level, the potential of such techniques to operational centres. In numerical weather forecasting, data assim- 
ilation has been implemented operationally very early: the European Centre for Medium-range Weather Forecast demonstrated that mathematical techniques, such as 4D-Var, could be used operationally with success on an even more complex system.

That is why an agency such as the IRSN is getting interested in the potential of data assimilation for their operations. This paper addresses with synthetic examples what a data assimilation system built on hypotheses as simple as possible, but without compromising the efficiency of the system (essentially the forecast skill), could achieve.

\subsection{State of the art}

Firstly, we would like to review the current status of inverse modelling and data assimilation of atmospheric tracer from an accidental origin, at continental scale. All the methods cited below are Bayesian in nature, and that is why they should not fundamentally yield different results.

Many of published inverse modelling techniques in atmospheric tracer source inversion, are of nonparametric nature: they attempt to retrieve large spatial fields of variables, rather than a few selected parameters. One typically looks for a source field, usually multidimensional, typically one dimension for time and two dimensions for the ground. If one knows the location of the source, then a one-dimensional time rate profile is usually sought. Robertson and Langner (1997); Seibert (2001) were among the first to attempt such an inversion using real data of the European Tracer Experiment (Nodop et al., 1998) (ETEX). Issartel and Baverel (2003); Issartel (2003) used projection methods (that are equivalent to a 4D-Var approach in a linear model context) and demonstrated the interest of a simplex method on synthetic data case inspired from ETEX. Bocquet (2005a) suggested to use a maximum entropy on the mean inference in order to implement additional constraints on the source such as positivity and boundedness. It was used successfully on the real ETEX data (Bocquet, 2005b, 2007; Krysta et al., 2008), on the Algeciras dispersion incident (Krysta and Bocquet, 2007) and on the Chernobyl accident (Davoine and Bocquet, 2007).

An advantage of these non-parametrical methods is the robustness that often stems from the convexity of the underlying cost functions. Another advantage is their immediate generalisation to more complex problems, such as multiple sources or source with a significant duration. They also share common grounds with other atmospheric chemistry problems such as ozone precursors source inversion in air pollution modelling or greenhouse gas fluxes inversion. Being deterministic methods, their aim is to provide an estimate of the source, but second-order sensitivity analysis is also possible (Bocquet, 2008).

In the case of the inversion of a point-wise accidental atmospheric source, one could use parametrical methods instead (relying on a few parameters), by looking for the location, time and rate of the source. For instance, Yee et al. (2008) simply compute the marginal posterior probability density function ( $\mathrm{pdf}$ ) on a few selected parameters on the ETEX experiment. Delle Monache et al. (2008) apply Markov Chain Monte Carlo techniques for a stochastic estimation of the Algeciras source location. The major advantage of these methods is the limited number of parameters that are retrieved. Owning to the small number of parameters, the full pdf (or marginal pdf) of these parameters can be retrieved.

Assimilation of observations for the forecast of accidental plume dispersion has been investigated in Politis and Robertson (2004); Bocquet (2007) on the ETEXI case, and on a hypothetical regional case around a power plant in Abida and Bocquet (2009). It was shown that the plume can efficiently be controlled because of the non-chaoticity of dispersion dynamics, provided the source is well retrieved.

\subsection{Objectives and outline}

The objective of this paper is to assess the performance of a simple inverse modelling and data assimilation system. In particular one is interested in not only the average performance of such a tool, but also in cases where it may fail, which is of utmost importance toward an implementation in operational centres. Different situations (sources, monitoring network, dispersion tools) of two different countries, France and Finland, are explored.

The setup of the data assimilation experiments, along with the models that are employed are described in Section 2. An efficient data assimilation system, common to the two national contexts, is presented in the same section. Although the methodology is simple, it differs from simpler least-square schemes, because it is assumed that the errors, being multiplicative, are source dependent.

Within the framework of Section 2, a sensitivity analysis is carried out in Section 3, varying the context: France or Finland, the meteorology for a fixed typical power plant, the power plants, the meteorological conditions, the monitoring network, etc. The data assimilation methodology assumes here that the origin of the radiological release, one of the power plants, is 
known. This should be realistic as the management of the nuclear power plant at risk should immediately raise an alert (to the national authority, possibly European Union, or the International Atomic Energy Agency). However, history has shown that this is not always the case. Besides, the accident may occur on a location abroad. It is therefore necessary to complement the operational data assimilation system with either a more complex system that does not assume that the location of the source is known, or with statistical tests that would assess the probability of a site to be responsible for the release. Since this paper is aimed at easily implementable tools, the focus is on this second option. Bayesian statistical tools are developed and tested in Section 4. 5 .

The conclusions and perspectives are given in Section

\section{Methods and setup}

\subsection{France and Finland setup}

As far as France is concerned, all the civil nuclear facilities are monitored, that is 19 nuclear power plants and a nuclear fuel reprocessing plant located at $\mathrm{La}$ Hague. The monitoring network that is used is the one that has been proposed by Saunier et al. (2009), called Descartes then, and renamed recently OperaAIR $(\alpha=1$ configuration as described in Saunier et al. (2009)). It is made of 100 stations, that measure activity concentrations over a range of $10^{-6}-10^{9} \mathrm{~Bq} \cdot \mathrm{m}^{-3}$.

As for Finland, it was chosen to monitor 6 sites. Two of them are located in Finland (one of them, Olkiluoto, potentially stands for two power plants as a third generation European pressurised reactor is under construction there). Two others are in Sweden and the last two ones are in Russia. The monitoring network is the actual "Uljas" Finnish ambient rate dose monitoring network of 255 stations. The power plant locations as well as the monitoring networks are represented in Fig. 1.

As a simplification, we have hypothesised that the stations of the Finnish and the French networks are measuring activity concentrations instead of ambient dose. This assumption simplifies the method since the assimilation of ambient dose would require a spatial model integration, and would require to properly take into account contamination effects of dose instruments.

The Finnish power plants are located on the shores, and the Swedish and Russian power plants are quite far from the Finnish monitoring network. This situation can lead to difficulties in the inverse modelling process. This is an important difference with the French setup where the monitored power plants cover the country land, even though not uniformly, and they are therefore well surrounded by the measuring stations.

\subsection{Dispersion modelling with PolaIr3D and SILAM}

The first numerical chemistry-transport model used in this study is PolaIR3D, the Eulerian model of the Polyphemus platform. As far as radionuclides are concerned, it has been validated on the European Tracer Experiment, on the Algeciras incident and on the Chernobyl accident (Quélo et al. (2007)).

After the gamma dose instruments, most of the current radionuclides monitoring devices are particle filters. That is why caesium-137, which disperses mostly as fine particulate matter, was chosen as the species of interest. The model integrates the concentration field $c$ of ${ }^{137} \mathrm{Cs}$, following the transport equation

$$
\frac{\partial c}{\partial t}+\operatorname{div}(\boldsymbol{u} c)=\operatorname{div}\left(\rho \boldsymbol{K} \nabla\left(\frac{c}{\rho}\right)\right)-\Lambda^{\mathrm{s}} c-\Lambda^{\mathrm{d}} c+\sigma
$$

where $\Lambda^{\mathrm{s}}$ is the wet scavenging rate, $\Lambda^{\mathrm{d}}$ represents the radioactive decay and $\sigma$ is the point-wise source for ${ }^{137} \mathrm{Cs}$. $\boldsymbol{K}$ is the matrix of turbulent diffusion, diagonal in practice. The vertical component is given by $K_{z}$, computed with Louis parametrisation (Louis, 1979). The horizontal component $K_{H}$ is taken null. Except for the boundary condition on the ground, all boundary conditions are taken null. The boundary condition on the ground is

$$
-K_{z} \nabla c \cdot \mathbf{n}=-v^{\text {dep }} c
$$

where $\mathbf{n}$ is the unitary vector upward oriented, and $v^{\text {dep }}$ is the dry deposition velocity of ${ }^{137} \mathrm{Cs}$.

The advection is implemented thanks to a third-order direct space-time scheme, with a Koren-Sweby flux limiter function. Because of the sharp gradients found, it is important that such a limiter be used. The diffusion scheme is integrated through an implicit second-order Rosenbrock scheme, with a three-point spatial scheme, and directional splitting.

Caesium-137 is modelled as a passive gaseous tracer with radioactive decay. Its half-life is 30 years. Its dry deposition is modelled by using a simple scheme with a constant deposition velocity: $v^{\text {dep }}=0.5 \mathrm{~cm} \mathrm{~s}^{-1}$. As far as the wet scavenging is concerned, the parametrisation used in this study is Belot of the form $\Lambda^{\mathrm{s}}=a p_{0}^{b}$, with $a=8 \times 10^{-5}$ and $b=0.8$. $p_{0}$ is the rain intensity, in $\mathrm{mm} / \mathrm{h}$ (Baklanov and Sørensen, 2001).

The SILAm dispersion model includes both Eulerian and Lagrangian dynamical cores. A description of the model and the Lagrangian core is given by Sofiev et al. 
(2006). This work uses the more recent Eulerian core, where horizontal and vertical advection is computed using the scheme by Galperin (2000). Vertical diffusion is discretised as described in Sofiev (2002) with the diffusion coefficient $K_{z}$ parametrised following Genikhovich et al. (2004).

Dry deposition is computed using the standard resistance analogy approach of Wesely (1989), with resistances for aerosols parametrised after Zhang et al. (2001). The wet deposition parametrisation follows Sofiev (2000) for gases and Sofiev et al. (2006) for aerosols.

Both transport models are then integrated in the European domain with a spatial resolution of $0.25^{\circ} \times 0.25^{\circ}$. The number of grid points in the domain simulation is $240 \times 160$ for both models. The PolaIR3D model is configured with 9 vertical levels ranging from 0 to 3300 $\mathrm{m}$, while 7 levels ranging up to $6300 \mathrm{~m}$ are used with Silam. Results are then saved respectively on French and Finnish subdomains. On these subdomains, defined as shown in Fig. 1, the indicators of performance concerning the ability of the system to forecast the radioactive plume will be calculated.

For this study, three periods of simulation are selected. The first one is the whole 2007 year, used to evaluate the performance of the system for one specific power plant, varying the meteorological conditions. Additionally, in order to test the average performance of the system on every power plant, two shorter periods (one week long) have been chosen. The first one is the week starting 2 December 2007, selected for its strong wind conditions. The second one is the week starting 8 June 2007, characterised by its weak wind speeds. The meteorological fields used in this study are the fields calculated by the operational model from the European Centre for Medium-range Weather Forecasts (ECMWF). They have a resolution of $0.25^{\circ} \times 0.25^{\circ}$ and are available every 3 hours.

\subsection{Inverse modelling methodology}

The observation equation

$$
\mu=H \sigma+\epsilon,
$$

encodes the source-receptor relation: $\boldsymbol{\mu} \in \mathbb{R}^{d}$ is the vector of activity concentration measurements; $\sigma \in \mathbb{R}^{N}$ is the vector that results from the discretisation of the source term; $\boldsymbol{\epsilon} \in \mathbb{R}^{d}$ is a vector that represents errors in the system (instrumental, representativity or model errors); $\boldsymbol{H} \in \mathbb{R}^{d \times N}$ is an operator, the Jacobian matrix, that not only stands for the observation operator but also the full dispersion model.
The Jacobian matrix can be computed column by column using the forward numerical model when the number of source parameters is smaller than the number of measurements. A column corresponds to the response of the system (a set of measurements in $\mathbb{R}^{d}$ ) to an elementary source, typically a point-wise instantaneous source with unit emission rate (read Abida and Bocquet (2009) for more details). Alternatively, it can be computed row by row when the number of source parameters is greater than the number of measurements. In this latter case, it is necessary to use the adjoint model since each row correspond to a solution of the adjoint of the numerical model, which can be seen as a drawback since deriving the adjoint can sometimes be a technological challenge. Equation (3) sets the inverse problem when one looks for the vector components of $\sigma$.

In order to compute each element of Eq. (3) with reliable, simple and efficient tools, with a view to operational constraints, we have made the following choices:

1. It is assumed that the source location is known and is one of the nuclear power plants that are monitored. This is consistent with the fact that in France and in Finland, the power plant management must report instantly any incident to the authorities.

2. Because the source location is known, we aim at retrieving the source rate profile. For a time resolution of one hour, this may represent of the order of a few hundreds of parameters to solve for (for instance 168 for a week of reconstruction).

3. This number of unknown parameters is small compared to the number of activity measurements that can be routinely obtained from the French or the Finnish monitoring networks. Thus, when posing the inverse problem, $\boldsymbol{H}$ should be computed column by column, using the forward model. This scheme avoids the derivation of the adjoint.

4. The computation of the columns or impulses, can be performed routinely. If the time resolution is set to one hour, operational centre would hourly compute one impulse for each one of the monitored power plants. It would be stored to build up $\boldsymbol{H}$. Note that these simulations can be trivially parallelised.

5. As a consequence, an up-to-date Jacobian matrix $\boldsymbol{H}$ should always be available.

The synthetic measurements $\boldsymbol{\mu}$ that are generated for the experiments contains errors $\boldsymbol{\epsilon}^{t}$ (called true errors) that are lognormally distributed:

$$
\boldsymbol{\mu}=\boldsymbol{H} \boldsymbol{\sigma}^{t}+\boldsymbol{\epsilon}^{t}
$$


with

$$
\epsilon_{i}^{t}=\left(e_{i}-1\right)\left[\boldsymbol{H} \boldsymbol{\sigma}^{t}\right]_{i}
$$

$\ln \left(e_{i}\right)$ being distributed according to $0.5 \times \mathcal{N}(0,1)$, the Gaussian distribution of mean 0 and standard deviation 0.5 . This implies that there is no bias in the median of $\boldsymbol{\epsilon}^{t}$. However the mean of $\boldsymbol{\epsilon}^{t}$ is biased. As far as amplitude is concerned, these errors are significant and realistic for an atmospheric tracer dispersion event. From this point, the true errors are unknown and even the distribution of the true errors is unknown. One has only access to the (noisy) observations $\boldsymbol{\mu}$. Nevertheless prior hypotheses can be made on the errors.

If the prior hypotheses on the errors used in the data assimilation scheme would follow the true errors (that have been generated with the lognormal distribution), then one should solve the inversion using a cost function that accounts for the prior hypothesis such as in Abida and Bocquet (2009).

But realistic errors do not have to follow a lognormal distribution law, and it would be unwise to suppose a priori that the errors are lognormal, even though the actual distribution might be similar. Here, we assume a priori that the errors are Gaussian, following a normal distribution

$$
p(\boldsymbol{\epsilon})=\frac{\exp \left(-\frac{1}{2} \boldsymbol{\epsilon}^{\mathrm{T}} \boldsymbol{R}^{-1} \boldsymbol{\epsilon}\right)}{\sqrt{(2 \pi)^{d}|\boldsymbol{R}|}},
$$

with error covariance matrix $\boldsymbol{R}$ (and $|\boldsymbol{R}|$ its determinant). From this point, elementary Bayes inference leads to the following cost function :

$$
\mathcal{L}(\boldsymbol{\sigma})=\frac{1}{2} \ln |\boldsymbol{R}|+\frac{1}{2}(\boldsymbol{H} \boldsymbol{\sigma}-\boldsymbol{\mu})^{\mathrm{T}} \boldsymbol{R}^{-1}(\boldsymbol{H} \boldsymbol{\sigma}-\boldsymbol{\mu}),
$$

where the first term, seemingly constant, will be at use shortly. At first, we are not assuming any regularisation of the inversion. Indeed, because $N \ll d$, no background term seems necessary, though this hypothesis will be discussed in section 3 .

Then, we assume a priori that the errors are Gaussian but of multiplicative nature: $\boldsymbol{R}$ is diagonal and the variances $[\boldsymbol{R}]_{i i}$ depend on the measurements $\mu_{i}$. Assuming that the variances are multiplicatively related to the true (errorless) measurements $\mu_{i}^{t}=\left[\boldsymbol{H} \boldsymbol{\sigma}^{t}\right]_{i}$ would be accurate but unfortunately an inversion crime, since one has only access to the noisy observations. Thus, a second idea is to relate the variances to the (noisy) observations $\mu_{i}$. But, we have proved heuristically (see Appendix B) and numerically checked that, on the assumption that the true errors are lognormal, this would lead to a large underestimation of the source by a factor of $\exp \left(-\frac{3}{2} \chi\right)$, where $\chi$ is the variance parameter of the lognormal distribution. In an accidental context, one must avoid such an undershoot.

As a consequence, it is assumed that the error attached to the measurement $\mu_{i}$ is proportional to the corresponding analysed measurement $[\boldsymbol{H \sigma}]_{i}$, which is the best approximation of $\mu_{i}$ that can be known: $\epsilon_{i} \sim$ $r[\boldsymbol{H} \boldsymbol{\sigma}]_{i} \mathcal{N}(0,1)$, with $r$ chosen to be $r=50 \%$. This also accounts for the fact that prior error distribution can only ideally match the true distribution. With this formulation, one obtains the following cost function:

$$
\mathcal{L}(\boldsymbol{\sigma})=\sum_{i=1}^{d}\left(\ln \left([\boldsymbol{H} \boldsymbol{\sigma}]_{i}\right)+\frac{1}{2 r^{2}} \frac{\left([\boldsymbol{H} \boldsymbol{\sigma}]_{i}-\mu_{i}\right)^{2}}{[\boldsymbol{H} \boldsymbol{\sigma}]_{i}^{2}}\right) .
$$

The retrieval of the source term $\sigma$ is carried out through the minimisation of the cost function Eq. (8), using the L-BFGS-B limited-memory quasi-Newton minimiser (Liu and Nocedal (1989)). More generally the online estimation of error covariances for atmospheric problems has been dicussed by Dee (1995).

\subsection{Data assimilation methodology}

In an emergency situation, and at current time $t_{n}$ the sequential data assimilation system counts three main steps :

1. Preprocessing step: All available data in the interval $\left[t_{n}-\Delta t_{a}, t_{n}\right]$ are collected (where $\Delta t_{a}$ is the assimilation interval). In parallel, the elementary solutions corresponding to this time interval are computed using the forward chemistrytransport model. The system needs to compute $\Delta t_{a} / \Delta t_{\text {source }}$ elementary solutions and store them (where $\Delta t_{\text {source }}$ is the reconstructed source resolution).

2. Analysis step: The updated $\boldsymbol{H}$ matrix is computed column-by-column using all elementary solutions from $t_{0}$ to $t_{n}$. The measurement vector $\boldsymbol{\mu}_{n}$ containing all the observations from $t_{0}$ to $t_{n}$ is also built up. Then an estimation of the source term $\sigma_{n}$ is obtained by minimisation of the cost function Eq. (8).

3. Forecast step: A forecast of $\Delta t_{f}$ hours from $t_{n}$ to $t_{n}+\Delta t_{f}$ is performed using the chemistry-transport model from $t_{0}$ and $t_{n}+\Delta t_{f}$. The source term used in this simulation is $\sigma_{n}$ between $t_{0}$ to $t_{n}$ and an estimation of the source rate between current time $t_{n}$ and $t_{n}+\Delta t_{f}$. We chose to implement the persistence assumption, which means that the source rate beyond current time is taken equal to the last retrieved component of $\sigma_{n}$. It is usually safer because for a decreasing rate profile, it will overestimate the source term rather than underestimate it. 
This can be invalidated by the presence of a second peak in the source term as we shall see.

This simple procedure has been tested successfully by Abida and Bocquet (2009), with a view to the targeting of observations, but at a regional scale rather than at continental scale. More details can be found there.

\subsection{The source term}

The source term that has been selected, provided by the IRSN, corresponds to a core-meltdown without hull breach. There is a second peak 24 hours after the beginning of the accident due to an intentional release to alleviate the pressure inside the confinement hull. The source term is displayed in Fig. 2, among further results.

\section{Sensitivity analysis of the data assimilation sys- tem}

To compare the reference and the retrieved field (the source rate profile or the concentration field of the plume), we use several statistical indicators. For the plume, a specific figure of merit indicator $\mathrm{fm}$ is defined at some time $\tau$ as follows:

$$
\mathrm{fm}=\frac{\sum_{h \in S} \min \left([\overline{\boldsymbol{c}}]_{h},\left[\boldsymbol{c}^{t}\right]_{h}\right)}{\sum_{h \in S} \max \left([\overline{\boldsymbol{c}}]_{h},\left[\boldsymbol{c}^{t}\right]_{h}\right)},
$$

where $S$ denotes the set of spatial grid cells index, $\boldsymbol{c}^{t}$ is the true concentration field at time $\tau$, and $\bar{c}$ represents the reconstructed plume field at the same time. It has been used in a similar context but in the ETEX context by Bocquet (2007).

The root mean square error (rmse) compares the source term reference and the estimate of the source at some time $\tau$ and is given by:

$$
\text { rmse }=\sqrt{\frac{1}{N} \sum_{n=1}^{N}\left(\left[\boldsymbol{\sigma}^{t}\right]_{n}-[\bar{\sigma}]_{n}\right)^{2}},
$$

where $\sigma^{t}, \bar{\sigma}$ are respectively the source reference and the estimated source at time $\tau$. A Pearson correlation coefficient is also used:

$$
\rho=\sum_{n=1}^{N} \frac{[\overline{\boldsymbol{\sigma}}-\langle\overline{\boldsymbol{\sigma}}\rangle]_{n}\left[\boldsymbol{\sigma}^{t}-\left\langle\boldsymbol{\sigma}^{t}\right\rangle\right]_{n}}{\sqrt{\left(\sum_{n=1}^{N}[\overline{\boldsymbol{\sigma}}-\langle\overline{\boldsymbol{\sigma}}\rangle]_{n}^{2}\right)\left(\sum_{n=1}^{N}\left[\boldsymbol{\sigma}^{t}-\left\langle\boldsymbol{\sigma}^{t}\right\rangle\right]_{n}^{2}\right)}},
$$

where \langle\rangle denotes the average over time.

\subsection{Time-averaged performance for one power plant}

To demonstrate the performance of the data assimilation system, the focus is first on the Belleville nuclear power plant which is central in France. Over one year of meteorology (2007), an accident was considered every hour. These 8760 accidents required as many sevenday simulations with PolaIR3D. For each accident, the source term was estimated by inverse modelling using all available observations (during one week, i.e. 168 hours), and the methodology described earlier. Averaging over all accidents, the mean reconstructed source is reported in Fig. 2, along with the true source. The dotted curves are the standard deviation lower and upper limits around the mean solution. The main estimation errors occur in the vicinity of the peaks. Yet it was checked that these errors are attenuated by the use of the objective function Eq. (8) that accounts for multiplicative errors.

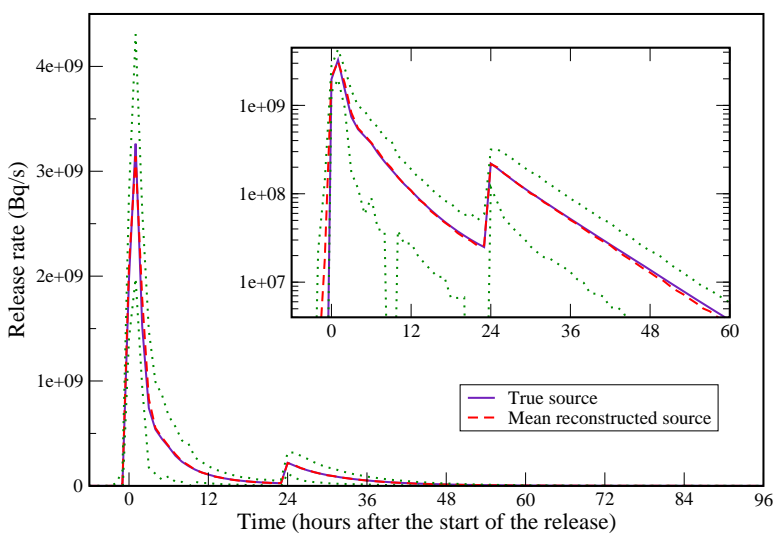

Figure 2: Mean reconstructed source (dashed line) as compared to the true source (full line) using all available observations. The upper and lower curves indicate the standard deviation range of the reconstructed sources. In insert, the same curves are represented using a logarithmic scale.

In an emergency situation, the observations are assimilated sequentially and an estimate of the source term is produced every hour. This is different from the previous a posteriori estimation which relies on all observations. Figure 3 reports the average sequential rmse on the source reconstruction. No impact of the second peak is visible on the curves because they are mainly driven by the errors on the first peak. The Pearson correlation coefficient is also reported. Obviously, the latter is not very stringent, and one should rather rely on rmse.

As for the plume forecast estimation, the figure of merit of the concentration field 3 hours after the assimilation time is plotted in Fig. 4. The impact of the uncer- 


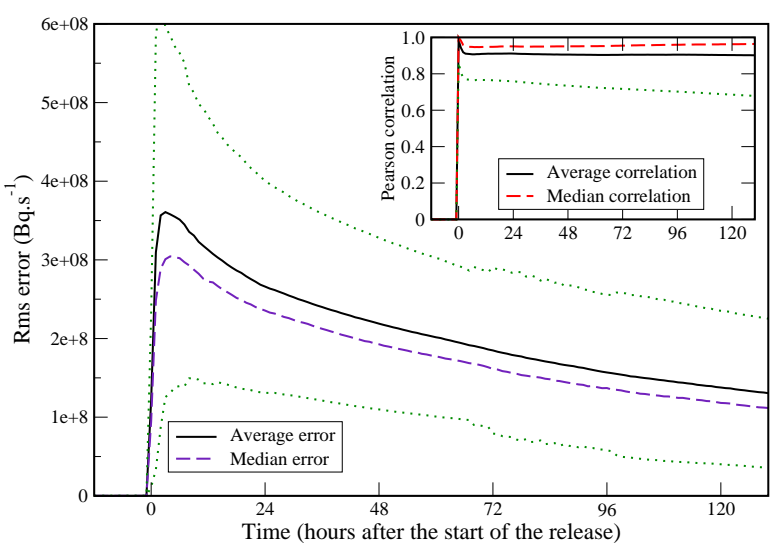

Figure 3: Mean (full line) and median (dashed line) root mean square error, assimilating observations sequentially. The upper and lower curves (dotted lines) indicate the standard deviation range of the average rmse. In inset, the Pearson correlation coefficient is displayed with a full line for the mean value and a dashed line for the median value. The mean correlation plus/minus the standard deviations are represented by the dotted curves.

tainty related to the second peak is patent with a drop of performance over four hours.

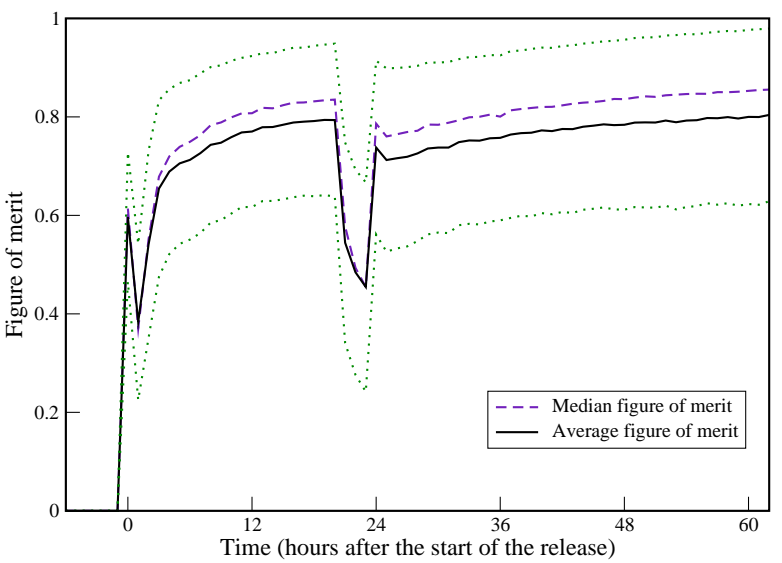

Figure 4: Mean (full line) and median (dashed line) figure of merit. The upper and lower curves (dotted lines) indicate the standard deviation range of the average figure of merit.

\subsection{Global average performance}

In the next step, the performance of the data assimilation system is evaluated through a more global systematic test. For the French and the Finnish context, all the power plants ( 20 in the French context and 6 in the Finnish one) is tested, over two different periods of 168 accidents (one accident every hour during one week).
The first period is one week in June 2007, chosen for its weak wind situation. The second period is one week in December 2007, with a strong wind situation. Like in Section 3.1, averaged performance are investigated for the source reconstruction and the forecast plume. For both periods, the results are similar to those obtained for the Belleville power plant over a whole year (same order of magnitude and evolution for the RMSE, correlations and figures of merit). The median solution is always better than the mean one, and the performance of the system are higher in the French context than in the Finnish one. This indicates that the system is mostly efficient, except in some situations where it completely fails (around $5 \%$ of the situations). These failure situations are associated to power plants located on borders or totally outside the monitoring network convex envelop.

\subsection{Usefulness of a source term regularisation}

In some cases, especially in the Finnish context, with monitored power plants near the shores or abroad, it was found that the inversion (through the minimisation of the cost function) may fail. This happens when the monitoring network has little overlap with the plume. It is then necessary to apply a regularisation, to use background information in the terminology of data assimilation. Some prior knowledge on the source term is hypothesised. For instance, simple prior statistics on the source is given by the Gaussian multivariate distribution

$$
p(\boldsymbol{\sigma})=\frac{\exp \left(-\frac{1}{2} \boldsymbol{\sigma}^{\mathrm{T}} \boldsymbol{B}^{-1} \boldsymbol{\sigma}\right)}{\sqrt{(2 \pi)^{N}|\boldsymbol{B}|}},
$$

where $\boldsymbol{B}$ is the background error covariance matrix, which will be taken diagonal in this context. The diagonal elements $[\boldsymbol{B}]_{i i}$ represent the a priori variances of the source, characterising some uncertainty on the source

$$
[\boldsymbol{B}]_{i j}=\mathrm{E}\left[\sigma_{i} \sigma_{j}\right],
$$

for all $i, j=1, \cdots, N$.

The solution of the inverse problem is then the minimum $\bar{\sigma}$ of the cost function

$$
\begin{aligned}
\mathcal{L}(\boldsymbol{\sigma})= & \frac{1}{2} \ln |\boldsymbol{R}|+\frac{1}{2}(\boldsymbol{\mu}-\boldsymbol{H} \boldsymbol{\sigma})^{\mathrm{T}} \boldsymbol{R}^{-1}(\boldsymbol{\mu}-\boldsymbol{H} \boldsymbol{\sigma}) \\
& +\frac{1}{2} \sigma^{\mathrm{T}} \boldsymbol{B}^{-1} \boldsymbol{\sigma} .
\end{aligned}
$$

$\boldsymbol{B}$ can simply be taken proportional to the identity $\boldsymbol{B}=$ 
$m^{2} \boldsymbol{I}$ and then the cost function can be written as :

$$
\begin{aligned}
\mathcal{L}(\sigma)= & \sum_{i=1}^{d}\left(\ln \left([\boldsymbol{H} \boldsymbol{\sigma}]_{i}\right)+\frac{1}{2 r^{2}} \frac{\left([\boldsymbol{H} \boldsymbol{\sigma}]_{i}-\mu_{i}\right)^{2}}{[\boldsymbol{H} \boldsymbol{\sigma}]_{i}^{2}}\right) \\
& +\frac{1}{2} \sum_{i=1}^{N} \frac{\sigma_{i}^{2}}{m^{2}}
\end{aligned}
$$

The value of scale parameter $m$ matters, because it determines the scale of the fluctuations in the source that should be considered relevant or not. A very rough estimation is given by the maximum rate of the source. It depends on the type of accident one is monitoring and a lower one should be chosen for a less critical accident, such as a breakdown of a vapour generating tube, with a preventive release into the atmosphere of overpressure.

In our case one has $m \simeq 10^{9} \mathrm{~Bq} / \mathrm{s}$. A too large value of $m$ would not render the regularisation operative for the critical cases (nevertheless it would not render the inversion impossible for the cases where regularisation is not necessary) whereas a too low value might smooth out relevant part of the source with high rate values that should have been reconstructed otherwise.

The scalar $m$ could be determined objectively using hyper-parameter estimation techniques (e.g. Davoine and Bocquet (2007); Krysta et al. (2008) in a similar context). But its use in operational systems remains risky, since the result of this estimation is very sensitive to the context and is also computationally demanding.

As an example let us consider the nuclear power plant of Kalinin (in Russia) in a strong wind case. Without regularisation the inversion fails, whereas it is quite successful when a background term is added, as reported in Fig. 5. In this case, both observations generated by PoLAIR3D and SILAm are employed. The two retrievals are consistent.

\section{Bayesian tests for the discrimination of sources}

Because one cannot always trust the nuclear facility management to report an accident immediately and because one cannot guarantee that an international alert will be raised without delay, one needs a diagnostic tool that does not assume that the location of the accident is known. Since we aim at an easily implementable tool, more academic methods that seek for the location are excluded, whatever successful they could have been on a few test-cases. Instead, we have developed Bayesian tests to help decide which nuclear site could be at the origin of the radiological plume.

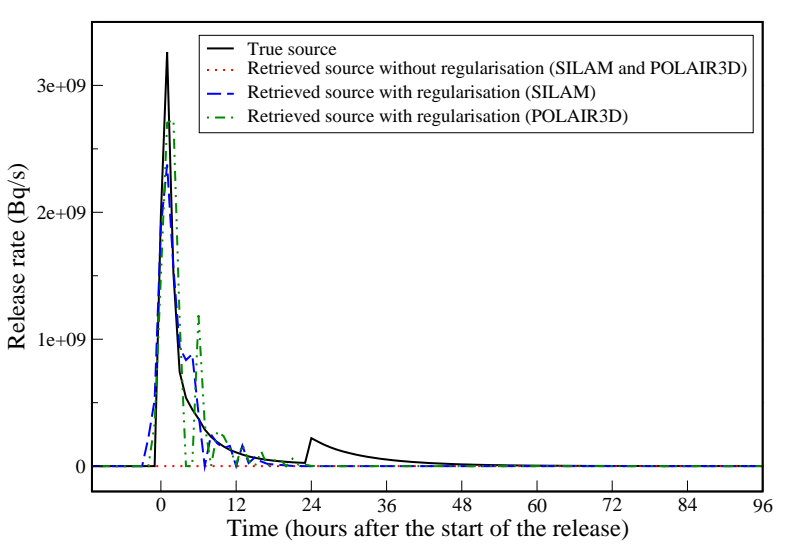

Figure 5: Impact of the regularisation in the extreme case of an inversion at Kalinin. The full line represents the true source. The retrieved source without regularisation using observations generated from PoLAIR3D or SILAM are null. The retrieved sources obtained with a regularised inversion are plotted with a dashed line (SILAM) and a dasheddotted line (PolaIR3D).

\subsection{Bayesian test with Gaussian assumptions}

As a first example, it is assumed that the prior statistics on the source follows the Gaussian distribution Eq. (12). This may be considered as a rough approximation since the source should not be allowed to have negative values. Yet, this simple assumption is often used in atmospheric dispersion inverse modelling with reasonable outcomes (Issartel and Baverel, 2003), although non-Gaussian assumptions are preferable (Bocquet, 2008).

The source-receptor relationship is given again by Eq. (3). Taking into account this mild prior information on the source, the likelihood of the measurements is

$$
p(\boldsymbol{\mu})=\int p(\boldsymbol{\sigma}) p(\boldsymbol{\mu} \mid \boldsymbol{\sigma}) \mathrm{d} \boldsymbol{\sigma},
$$

where $\mathrm{d} \sigma$ represent the integration element over all $\sigma_{k}$ control variables. The conditional density of the dataset knowing the source $p(\boldsymbol{\mu} \mid \boldsymbol{\sigma})$, is easily given by the errors

$$
p(\boldsymbol{\mu} \mid \boldsymbol{\sigma})=\frac{\exp \left(-\frac{1}{2}(\boldsymbol{\mu}-\boldsymbol{H} \boldsymbol{\sigma})^{\mathrm{T}} \boldsymbol{R}^{-1}(\boldsymbol{\mu}-\boldsymbol{H} \boldsymbol{\sigma})\right)}{\sqrt{(2 \pi)^{d}|\boldsymbol{R}|}},
$$

where $\boldsymbol{R} \in \mathbb{R}^{d \times d}$ is the observation error covariance matrix. To simplify the integration, the error attached to measurement $\mu_{i}$ is no longer assumed proportional to the corresponding analysed measurement, but to the measurement itself: $\epsilon_{i} \sim r \mu_{i} \mathcal{N}(0,1)$. It was explained earlier that it may lead to a severe under-estimation of the source if for instance the real errors follow a lognormal distribution. But source estimation is not the point 
here, and this approximation is secondary for these tests. The Gaussian integration in Eq. (16) on $\sigma$ then leads to (see Appendix A for details)

$$
p(\boldsymbol{\mu})=\frac{\exp \left(-\frac{1}{2} \boldsymbol{\mu}^{\mathrm{T}}\left(\boldsymbol{H} \boldsymbol{B} \boldsymbol{H}^{\mathrm{T}}+\boldsymbol{R}\right)^{-1} \boldsymbol{\mu}\right)}{\sqrt{(2 \pi)^{d}\left|\boldsymbol{H} \boldsymbol{B} \boldsymbol{H}^{\mathrm{T}}+\boldsymbol{R}\right|}} .
$$

The likelihood $p(\boldsymbol{\mu})$ represents the probability of observing $\boldsymbol{\mu}$ assuming that the statistical distribution of the source follows Eq. (12). If one knows the location of the release, say nuclear site $i$, then it is straightforward to obtain the likelihood of the dataset $\boldsymbol{\mu}$ provided the source prior statistics are Gaussian, and that the source is located at site $i$. The Jacobian matrix $\boldsymbol{H}$ can be replaced by that Jacobian matrix of site $i$ (a submatrix of $\boldsymbol{H})$.

$$
p_{i}(\boldsymbol{\mu})=\frac{\exp \left(-\frac{1}{2} \boldsymbol{\mu}^{\mathrm{T}}\left(\boldsymbol{H}_{i} \boldsymbol{B} \boldsymbol{H}_{i}^{\mathrm{T}}+\boldsymbol{R}\right)^{-1} \boldsymbol{\mu}\right)}{\sqrt{(2 \pi)^{d}\left|\boldsymbol{H}_{i} \boldsymbol{B} \boldsymbol{H}_{i}^{\mathrm{T}}+\boldsymbol{R}\right|}} .
$$

$\mathcal{L}=\frac{1}{2} \boldsymbol{\mu}^{\mathrm{T}}\left(\boldsymbol{H}_{i} \boldsymbol{B} \boldsymbol{H}_{i}^{\mathrm{T}}+\boldsymbol{R}\right)^{-1} \boldsymbol{\mu}$, in the exponential of Eq. (19) has been shown by Bocquet (2008) to be an objective measure of the gain of information by the observations $\boldsymbol{\mu}$ with respect to the hypothesis that the source is at site $i$. Intuitively, the bigger this information, the smaller the likelihood of the measurements to be explained by a source at site $i$. It is straightforward to check that this is indeed the case on Eq. (19).

As a consequence, it is possible to use the set of $p_{i}$, with $i$ running on all the suspected release sites. The sum $\sum_{i} p_{i}$, does not need to be 1 , but the $p_{i}$ can be normalised by their sum. For practical purposes, since the inversion of the matrix $\boldsymbol{H}_{i} \boldsymbol{B} \boldsymbol{H}_{i}^{\mathrm{T}}+\boldsymbol{R}$ in $\mathbb{R}^{d \times d}$ can be expensive, the Sherman-Morrison-Woodbury transform can be used to invert the matrix $\boldsymbol{H}_{i}^{\mathrm{T}} \boldsymbol{R}^{-1} \boldsymbol{H}_{i}+\boldsymbol{B}^{-1}$ in $\mathbb{R}^{N \times N}$ $(N \ll d)$ instead, so as to speed up the computation of each $p_{i}(\mu)$. Then the $p_{i}$ would represent normalised indices assessing the likelihood of each site, on the condition that the release took place on one of the predetermined sites.

When the latter assumption is violated, the normalized $p_{i} /\left(\sum_{j} p_{j}\right)$ are meaningless but the absolute low values of all $p_{i}$ should reveal this violation.

\subsection{Bayesian tests knowing the source term shape}

The Gaussian prior Eq. (12) is not very constraining. For instance, each one of the source independent scalar rates could either be positive or negative. On the other hand, operational centres have built a list of possible source terms for several types of accidents. As a prior piece of information, one could assume that the source term (say $\sigma_{b}$ ) belongs to this list. However it is unlikely that the magnitude of the real event would match the magnitude of this prior, even though the shape of the source rates is similar. Thus, one could rather assume that the source is of the form $\lambda \sigma_{b}$, with $\lambda$ a positive parameter distributed according to some given law $p(\lambda)$.

The prior distribution of the source is then formally given by

$$
p(\sigma)=\int_{0}^{\infty} \delta\left(\sigma-\lambda \sigma_{b}\right) p(\lambda) \mathrm{d} \lambda .
$$

As a result the observations likelihood reads

$$
p(\boldsymbol{\mu})=\int p(\boldsymbol{\sigma}) p(\boldsymbol{\mu} \mid \boldsymbol{\sigma}) \mathrm{d} \boldsymbol{\sigma}=\int_{0}^{\infty} p(\lambda) p\left(\boldsymbol{\mu} \mid \lambda \boldsymbol{\sigma}_{b}\right) \mathrm{d} \lambda .
$$

For instance, $\lambda$ could follow a gamma distribution

$$
p(\lambda)=\frac{\lambda^{k-1} e^{-\lambda / \theta}}{\theta^{k} \Gamma(k)}
$$

In the case ( $k=1$, exponential law), the likelihood reads (see Appendix $\mathrm{C}$ for details)

$$
\begin{aligned}
p(\boldsymbol{\mu})= & \sqrt{\frac{\pi}{2}} \frac{e^{-\frac{1}{2} \boldsymbol{\mu}^{\mathrm{T}} \boldsymbol{R}^{-1} \boldsymbol{\mu}}}{\theta \sqrt{(2 \pi)^{d}|\boldsymbol{R}|}} \frac{e^{\frac{\left(\mu^{\mathrm{T}} \boldsymbol{R}^{-1} \mu_{b}-\theta^{-1}\right)^{2}}{2 \mu_{b} \boldsymbol{R}^{-1} \mu_{b}}}}{\sqrt{2 \boldsymbol{\mu}_{b}^{\mathrm{T}} \boldsymbol{R}^{-1} \boldsymbol{\mu}_{b}}} \times \\
& {\left[1-\Phi\left(\frac{\theta^{-1}-\boldsymbol{\mu}^{\mathrm{T}} \boldsymbol{R}^{-1} \boldsymbol{\mu}_{b}}{\sqrt{2 \boldsymbol{\mu}_{b}^{\mathrm{T}} \boldsymbol{R}^{-1} \boldsymbol{\mu}_{b}}}\right)\right], }
\end{aligned}
$$

where $\Phi(u)=\frac{2}{\sqrt{\pi}} \int_{0}^{u} e^{-x^{2}} \mathrm{~d} x$ is the error function, and $\boldsymbol{\mu}_{b}=\boldsymbol{H} \boldsymbol{\sigma}_{b}$ is the errorless set of observations that would be obtained from the first guess source term $\sigma_{b}$. This case favours low scale factor $\lambda$. Setting $\theta=1$ implies that the average source term is $\sigma_{b}$.

A more appropriate prior distribution of $\lambda$ could be a gamma distribution of index $k=2$ which excludes too low values for $\lambda$, and reaches a maximum at $\theta$ (the mean being $2 \theta$ ). The likelihood reads

$$
\begin{aligned}
& p(\boldsymbol{\mu})=\frac{e^{-\frac{1}{2} \boldsymbol{\mu}^{\mathrm{T}} \boldsymbol{R}^{-1} \boldsymbol{\mu}}}{\theta^{2} \sqrt{(2 \pi)^{d}|\boldsymbol{R}|}} \frac{1}{\boldsymbol{\mu}_{b}^{\mathrm{T}} \boldsymbol{R}^{-1} \boldsymbol{\mu}_{b}} \times \\
& \left\{1-\sqrt{\frac{\pi}{2}} \frac{\theta^{-1}-\boldsymbol{\mu}^{\mathrm{T}} \boldsymbol{R}^{-1} \boldsymbol{\mu}_{b}}{\sqrt{\boldsymbol{\mu}_{b}^{\mathrm{T}} \boldsymbol{R}^{-1} \boldsymbol{\mu}_{b}}}\left[1-\Phi\left(\frac{\theta^{-1}-\boldsymbol{\mu}^{\mathrm{T}} \boldsymbol{R}^{-1} \boldsymbol{\mu}_{b}}{\sqrt{2 \boldsymbol{\mu}_{b}^{\mathrm{T}} \boldsymbol{R}^{-1} \boldsymbol{\mu}_{b}}}\right)\right]\right\} .
\end{aligned}
$$

In order for the average source term to be $\sigma_{b}$, one could choose in this case $\theta=1 / 2$. But $\theta=1$ is preferable 
because it would set the mode of the distribution to $\lambda=$ 1 and hence to the source term $\sigma_{b}$.

Lastly, a semi-Gaussian distribution of density $p(\lambda)=$ $\sqrt{\frac{2}{\pi}} e^{-\frac{\lambda^{2}}{2 \theta}}$, would yield

$$
\begin{gathered}
p(\boldsymbol{\mu})=\frac{e^{-\frac{1}{2} \boldsymbol{\mu}^{\mathrm{T}} \boldsymbol{R}^{-1} \boldsymbol{\mu}}}{\sqrt{\theta} \sqrt{(2 \pi)^{d}|\boldsymbol{R}|}} \frac{e^{\frac{\left(\mu^{\mathrm{T}} \boldsymbol{R}^{-1} \mu_{b}\right)^{2}}{2\left(\theta^{-1}+\boldsymbol{\mu}_{b}^{\mathrm{T}} \boldsymbol{R}^{-1} \mu_{b}\right)}}}{\sqrt{\theta^{-1}+\boldsymbol{\mu}_{b}^{\mathrm{T}} \boldsymbol{R}^{-1} \boldsymbol{\mu}_{b}}} \times \\
{\left[1+\Phi\left(\frac{\boldsymbol{\mu}^{\mathrm{T}} \boldsymbol{R}^{-1} \boldsymbol{\mu}_{b}}{\sqrt{2\left(\theta^{-1}+\boldsymbol{\mu}_{b}^{\mathrm{T}} \boldsymbol{R}^{-1} \boldsymbol{\mu}_{b}\right)}}\right)\right] .}
\end{gathered}
$$

One may investigate the skills of these Bayesian tests where the shape of $\sigma_{b}$ is the one of the true source but with the wrong scale $\sigma_{b}=\lambda_{t} \sigma_{t}$, since it is quite difficult to guess a priori the right scale. In that case defining $\|\boldsymbol{\mu}\|_{\boldsymbol{R}^{-1}}^{2}=\boldsymbol{\mu}^{\mathrm{T}} \boldsymbol{R}^{-1} \boldsymbol{\mu}, p(\boldsymbol{\mu})$ only depends on the norm $\left\|\mu_{b}\right\|_{\boldsymbol{R}^{-1}}$, and on the true scale parameter $\lambda_{t}$.

\subsection{Validation}

The following validation of the tests has been carried out for both Polair3D and SiLAM.

\subsubsection{Performance with time}

At first we choose $m=10^{9} \mathrm{~Bq} / \mathrm{s}$ for the Gaussian test and $\lambda_{t}=1$ for the other (non-Gaussian) Bayesian tests, which corresponds to optimal conditions for these tests. We implement these new Bayesian tests on the case of Sosnovy Bor. The (synthetic) true accident originates from this plant and the likelihood of Sosnovy Bor, Loviisa, Olkiluoto, Kalinin, Oskarshamn, and Forsmark to be the source is computed at $1,2, \ldots, 7$ hours after the beginning of the accident. The results for the Gaussian test are displayed in panel (a) of Fig. 6. The results for the other Bayesian tests look very similar but they manage to discriminate the correct release site faster than the Gaussian test (typically 2 hours faster).

A much more tangential case, with a poorly observed plume, is given by a fictitious accident occurring at Kalinin. In that specific example, the Gaussian Bayesian test fails to discriminate Kalinin from the other sites mentioned above. However the three other non-Gaussian tests managed to do so gradually, as reported in panels (b), (c) and (d) of Fig. 6. They almost perform equally well, with an understandable asset for the tests that have knowledge of the rate profile, up to a scalar factor.

\subsubsection{Changing the prior fluctuation scale}

As mentioned earlier, the prior scale of the source should be chosen wisely depending on the expected type of accident, and the order of magnitude of the source term. We have studied the performance of these Bayesian tests when the scales $m$ or $\lambda_{t}$ are varied and deviate from the true scale.

One typical example is reported on Fig. (7), when Belleville is the source, and the 19 other French sites are tested. The curves showing the likelihood for three sites with prominent features (Belleville, Dampierre and Fessenheim) are shown 6 hours after the beginning of the accident. The Gaussian test is used. For very low values of $m$, the test cannot discriminate the nuclear power plants. For very high values of $m$, the test picks up the

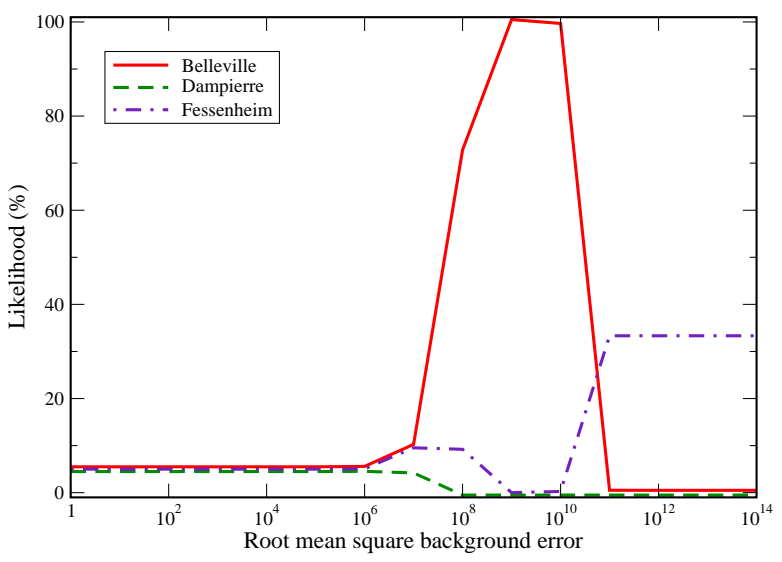

Figure 7: Gaussian Bayesian test for an accident in Belleville 6 hours after the start of the release. The root mean square of the background error which is the scale $m$ of the fluctuation of the hypothesised source, is varied and the test is performed for $\log _{10}(m)=0,1, \cdots, 14$.

wrong site: Fessenheim. Indeed, this site is farther away (on the French-German border) and a very large source is compatible with observations then, whereas the true source magnitude is several orders of magnitude different from the expected fluctuations thus leading to the exclusion of Belleville.

Thus, in this case, there is a range of validity for the test of four orders of magnitude.

In the case of the non-Gaussian tests, the behaviour for low values of $\lambda_{t}$ is the same, but the performance for high values of $\lambda_{t}$ are widely more robust, and the tests fail only for unrealistic high values of $\lambda_{t}$.

\subsubsection{Global performance of the Bayesian tests}

These indicators need to be tested on all potential sources, so as to estimate their global performance. In order to do so, the strong wind context, that is from 25 
November 2007 to 25 December 2007, is considered. Every hour an accident is considered on each one of the 20 nuclear sites in France. For each batch of $p=20$ accidents, a $p \times p$ matrix $\boldsymbol{L}$ is built for all subsequent times after the start of the accidents. The entry $[\boldsymbol{L}]_{i j}$ is defined by the likelihood of site $i$ in the accident from site $j$. Only the normalised trace, $\frac{1}{p} \operatorname{Tr}(\boldsymbol{L})$ is studied. It tells how well the correct site is identified, on average. A trace is computed for each time lag from the start of the release: $1 \mathrm{~h}, 2 \mathrm{~h}, \ldots$. Each one of them is averaged over the whole strong wind 10-day period. According to Krysta and Bocquet (2007); Abida and Bocquet (2009), we expect the performance to be weaker compared to a context with more stationary winds.

First, the Gaussian Bayesian test is used, with a $\boldsymbol{B}$ matrix proportional to the identity. Its proportionality coefficient is taken to be $m^{2}=M^{2} / \tau$, where $M$ is the total mass expected to be released, and $\tau$ is the number of one-hour time-steps after the start of the release. The non-Gaussian Bayesian tests have also been extensively tested. First the prior $\sigma_{b}$ is taken equal to the true source, which represents the most favourable case. Then these tests were carried out with priors $\sigma_{b}$ whose shapes were significantly different from the true source profile (for example taken as a monotonic or even as a constant term). The results are reported in Fig. 8(a) for the Gaussian tests, Fig. 8(b) for the semi-Gaussian tests, and Fig. 8(c) for the semi-Gaussian test with a wrong first guess. The average performances are impacted by outliers, but the skills of the median are excellent. These outliers are due to a poor observability of an event, that the swift dispersion of radionuclides by strong advection makes worse. The results are comparable for the Gaussian test and the non-Gaussian ones. Even when the prior $\sigma_{b}$ is different from the true source, the skill of the median still reaches almost $100 \%$. However, it does so later, typically 5 hours later. These results confirm the robustness of these non-Gaussian Bayesian tests.

\section{Conclusion}

In this paper, a semi-automatic sequential data assimilation system has been developed. In the event of an accidental atmospheric release of radionuclides from a nuclear power plant, the objective of such a system is mainly to accurately forecast the radioactive plume. This ability is strongly constrained by the knowledge of the source field and inverse modelling techniques have been used in this aim. The main concern of this paper was to use algorithms simple enough to be implemented in an operational context, but still state-of-the-art.
It was assumed that the source location is known soon enough. Consequently the Jacobian matrix can be computed efficiently using the forward dispersion numerical model, without resorting to the adjoint, and trivial parallelism can be used. Then the inversion of the sourcereceptor equation is performed with Gaussian assumptions for errors statistics distributions. Yet it is assumed that the variance of these errors are proportional to the activity concentration value at the observation site. At first, no regularisation and no background term has been used, though this is contemplated later.

To evaluate the performance of the system, synthetic experiments have been generated, varying the meteorological situations, the models, the monitoring network, the release locations, etc., based on the cases of France and Finland. The reconstruction statistical indicators show good results in average. The source is swiftly and well estimated and the forecast is accurate, even though the assumption of persistence for the source first guess can lead to a temporary (from 1 to 6 hours depending on the forecast time) deterioration in the forecast quality if the true source is not monotonic. Cases where the system failed (about $5 \%$ in our context) have been identified and alternate solutions have been proposed. The critical situations are all due to a lack of observability and concern mainly the power plants located on the periphery of the monitoring networks (near the frontiers, on the shores or abroad).

Aside from accounting for multiplicative errors, a second methodological novelty has been introduced. Bayesian statistical indicators have been developed and tested, when the origin of the release is not known or not soon enough. These tests determine the likelihood of a plant to be responsible for the release, knowing the observations and some prior ideas on the source term. They differ from each other by the assumptions made on the prior statistics on the source. The first indicator assumes a Gaussian distribution for these statistics. The results are very good in average, over a large range of value for the parameters in the prior. Yet, this indicator may fail in critical situations, for example for a release site far from the monitoring network. A second type of indicators have been proposed, assuming that the temporal profile of the source is known but not its exact magnitude. Then the results are excellent even when the prior temporal profile does not match the true one. The guilty power plant is quickly identified even when its location is far from the monitoring network.

Additional tests have been performed that are not reported here because they have not made systematic. For instance, what is result of the inversion for an uncharted source location? 
In this paper, it was assumed that the monitoring network were measuring directly activity concentrations in the air whereas current networks are mostly reporting gamma dose. Assimilating the gamma dose measurements using the proper integrating observation operator may amplify the potential errors, and this would need to be taken into account in a future operational system.

Acknowledgements. The authors thank R. Abida for sharing his expertise and interesting discussions. This paper is a contribution to the INSU/LEFE-ASSIM project 2007-21: "Application of advanced data assimilation techniques to the dispersion of accidental release of pollutants in case of emergency".

\section{Appendix A. Bayesian test with Gaussian assump- tion}

If the prior statistics of the source follows the Gaussian distribution Eq. (12), i.e.

$$
p(\boldsymbol{\sigma})=\frac{\exp \left(-\frac{1}{2} \boldsymbol{\sigma}^{\mathrm{T}} \boldsymbol{B}^{-1} \boldsymbol{\sigma}\right)}{\sqrt{(2 \pi)^{N}|\boldsymbol{B}|}},
$$

and since the conditional density of the dataset knowing the source is given by the errors (Eq. (17))

$$
p(\boldsymbol{\mu} \mid \boldsymbol{\sigma})=\frac{\exp \left(-\frac{1}{2}(\boldsymbol{\mu}-\boldsymbol{H} \boldsymbol{\sigma})^{\mathrm{T}} \boldsymbol{R}^{-1}(\boldsymbol{\mu}-\boldsymbol{H} \boldsymbol{\sigma})\right)}{\sqrt{(2 \pi)^{d}|\boldsymbol{R}|}},
$$

the likelihood of the measurements is given by Eq. (16)

$$
p(\boldsymbol{\mu})=\int p(\boldsymbol{\sigma}) p(\boldsymbol{\mu} \mid \boldsymbol{\sigma}) \mathrm{d} \boldsymbol{\sigma}
$$

Direct integration of Eq. (A.3) gives

$$
\begin{aligned}
p(\boldsymbol{\mu})= & \frac{\exp \left(-\frac{1}{2} \boldsymbol{\mu}^{\mathrm{T}} \boldsymbol{R}^{-1} \boldsymbol{\mu}\right)}{\sqrt{(2 \pi)^{d}|\boldsymbol{B} \| \boldsymbol{R}|} \times} \\
& \frac{\exp \left(\frac{1}{2} \boldsymbol{\mu}^{\mathrm{T}} \boldsymbol{R}^{-1} \boldsymbol{H}\left(\boldsymbol{H}^{\mathrm{T}} \boldsymbol{R}^{-1} \boldsymbol{H}+\boldsymbol{B}^{-1}\right)^{-1} \boldsymbol{H}^{\mathrm{T}} \boldsymbol{R}^{-1} \boldsymbol{\mu}\right)}{\sqrt{\left|\boldsymbol{H}^{\mathrm{T}} \boldsymbol{R}^{-1} \boldsymbol{H}+\boldsymbol{B}^{-1}\right|}}
\end{aligned}
$$

The Sherman-Morrison-Woodbury transform, applied to the numerator of the last member of the right hand side term in Eq. (A.4), gives

$$
\begin{aligned}
\left(\boldsymbol{H} \boldsymbol{B} \boldsymbol{H}^{\mathrm{T}}+\boldsymbol{R}\right)^{-1} & =\boldsymbol{R}^{-1}- \\
& \boldsymbol{R}^{-1} \boldsymbol{H}\left(\boldsymbol{H}^{\mathrm{T}} \boldsymbol{R}^{-1} \boldsymbol{H}+\boldsymbol{B}^{-1}\right)^{-1} \boldsymbol{H}^{\mathrm{T}} \boldsymbol{R}^{-1}
\end{aligned}
$$

and the determinant matrix lemma for the denominator gives

$$
\left|\boldsymbol{H} \boldsymbol{B} \boldsymbol{H}^{\mathrm{T}}+\boldsymbol{R}\right|=\left|\boldsymbol{H}^{\mathrm{T}} \boldsymbol{R}^{-1} \boldsymbol{H}+\boldsymbol{B}^{-1}\|\boldsymbol{B}\| \boldsymbol{R}\right|
$$

Thus Eq. (A.4) becomes

$$
p(\boldsymbol{\mu})=\frac{\exp \left(-\frac{1}{2} \boldsymbol{\mu}^{\mathrm{T}}\left(\boldsymbol{H} \boldsymbol{B} \boldsymbol{H}^{\mathrm{T}}+\boldsymbol{R}\right)^{-1} \boldsymbol{\mu}\right)}{\sqrt{(2 \pi)^{d}\left|\boldsymbol{H B} \boldsymbol{H}^{\mathrm{T}}+\boldsymbol{R}\right|}}
$$

The Eq. (A.4) and Eq. (A.7) are strictly equivalent. One can use either one equation or the other to retrieve the likelihood of the set of measurements $\boldsymbol{\mu}$ provided that the source is located at site i $p_{i}(\boldsymbol{\mu})$ by replacing all $\boldsymbol{H}$ by its submatrix $\boldsymbol{H}_{i}$ associated with site i. The interpretation seems more convenient with Eq. (A.7) since one retrieves in the exponential the term $\mathcal{L}=$ $\frac{1}{2} \boldsymbol{\mu}^{\mathrm{T}}\left(\boldsymbol{H}_{i} \boldsymbol{B} \boldsymbol{H}_{i}^{\mathrm{T}}+\boldsymbol{R}\right)^{-1} \boldsymbol{\mu}$, which has been shown by Bocquet (2008) to be an objective measure of the gain of information by the observations $\boldsymbol{\mu}$ with respect to the hypothesis that the source is at site $i$. Nevertheless, for practical purposes, when $N \ll d$, it is more convenient to implement Eq. (A.4), as the size of the matrix to invert (which is the critical process in the implementation) is much smaller.

\section{Appendix B. Source bias without online error esti- mation}

We assume that the true errors are lognormal. However, unaware of the exact distribution of the errors, the operator assumes normal obervational errors that are proportional to the measurements. The observational error covariance matrix $\boldsymbol{R}$ is diagonal with diagonal element $[\boldsymbol{R}]_{i i}=r^{2} \mu_{i}^{2}$, as explained in Section 2.3. Like for the whole paper, it is assumed that the number of observation is significantly greater than the number of emission rates to retrieve. Then the solution of the inverse problem Eq. (3) is

$$
\overline{\boldsymbol{\sigma}}=\left(\boldsymbol{H}^{\mathrm{T}} \boldsymbol{R}^{-1} \boldsymbol{H}\right)^{-1} \boldsymbol{H}^{\mathrm{T}} \boldsymbol{R}^{-1} \boldsymbol{\mu},
$$

Each matrix element of the denominator is of the form

$$
\left[\boldsymbol{H}^{\mathrm{T}} \boldsymbol{R}^{-1} \boldsymbol{H}\right]_{k l}=\sum_{i=1}^{d} r^{-2} \mu_{i}^{-2}[\boldsymbol{H}]_{i k}[\boldsymbol{H}]_{i l}
$$

By the definition of the synthetic experiment, the observation $\mu_{i}$ reads $x_{i} \mu_{i}^{t}$, where $\mu_{i}^{t}$ is the true observation, which cannot be accessed directly, and $x_{i}$ is a lognormal random draw. A heuristic argument is that with 
many observations for a limited rate variables, this entry averages out on the log-normal errors:

$$
\begin{aligned}
{\left[\boldsymbol{H}^{\mathrm{T}} \boldsymbol{R}^{-1} \boldsymbol{H}\right]_{k l} } & \simeq \mathrm{E}_{x}\left[\left[\boldsymbol{H}^{\mathrm{T}} \boldsymbol{R}^{-1} \boldsymbol{H}\right]_{k l}\right] \\
& \simeq \sum_{i=1}^{d} \mathrm{E}\left[x_{i}^{-2}\right]\left(r \mu_{i}^{t}\right)^{-2}[\boldsymbol{H}]_{i k}[\boldsymbol{H}]_{j l} .
\end{aligned}
$$

And, similarly for $\boldsymbol{H}^{\mathrm{T}} \boldsymbol{R}^{-1} \boldsymbol{\mu}$, one obtains

$$
\begin{aligned}
{\left[\boldsymbol{H}^{\mathrm{T}} \boldsymbol{R}^{-1} \boldsymbol{\mu}\right]_{k} } & \simeq \mathrm{E}_{x}\left[\left[\boldsymbol{H}^{\mathrm{T}} \boldsymbol{R}^{-1} \boldsymbol{\mu}\right]_{k}\right] \\
& \simeq \sum_{i=1}^{d} \mathrm{E}\left[x_{i}^{-1}\right] r^{-2}\left(\mu_{i}^{t}\right)^{-1}[\boldsymbol{H}]_{i k} .
\end{aligned}
$$

With a lognormal law of standard deviation parameter $\sigma$, one has $\mathrm{E}\left[x_{i}^{-2}\right] \propto \exp \left(2 \sigma^{2}\right)$, and $\mathrm{E}\left[x_{i}^{-1}\right] \propto$ $\exp \left(\sigma^{2} / 2\right)$. As a consequence, the retrieved source is

$$
\overline{\boldsymbol{\sigma}} \simeq e^{-\frac{3}{2} \sigma^{2}}\left(\boldsymbol{H}^{\mathrm{T}} \boldsymbol{R}_{t}^{-1} \boldsymbol{H}\right)^{-1} \boldsymbol{H}^{\mathrm{T}} \boldsymbol{R}_{t}^{-1} \boldsymbol{\mu}^{t},
$$

where $\boldsymbol{R}_{t}$ is the unbiased observation error covariance matrix that would have been used if one knew the true measurements. This explains the bias of $e^{-\frac{3}{2} \sigma^{2}}$ claimed in the text. Even though the argument is heuristic, this value has been confirmed by numerical experiments using the data assimilation system presented in this paper.

\section{Appendix C. Bayesian tests knowing the source term shape}

If we assume that the source term is of the form $\lambda \sigma_{b}$ with $\sigma_{b}$ being a known source shape and $\lambda$ a positive parameter distributed according to some given law $p(\lambda)$, the likelihood of the dataset of measurements $\boldsymbol{\mu}$ is given by Eq. (21)

$$
p(\boldsymbol{\mu})=\int_{0}^{+\infty} p(\lambda) p\left(\boldsymbol{\mu} \mid \lambda \boldsymbol{\sigma}_{b}\right) \mathrm{d} \lambda
$$

If $\lambda$ follows a gamma distribution $p(\lambda)=\frac{\lambda^{k-1} e^{-\frac{\lambda}{\theta}}}{\theta^{k} \Gamma(k)}$ with $k=1$ (in other words an exponential law), then this equation becomes

$$
\begin{aligned}
p(\boldsymbol{\mu})=\int_{0}^{+\infty} & \frac{\exp \left(-\frac{\lambda}{\theta}\right)}{\theta} \times \\
& \frac{\exp \left(-\frac{1}{2}\left(\lambda \boldsymbol{H} \boldsymbol{\sigma}_{b}-\boldsymbol{\mu}\right)^{\mathrm{T}} \boldsymbol{R}^{-1}\left(\lambda \boldsymbol{H} \boldsymbol{\sigma}_{b}-\boldsymbol{\mu}\right)\right)}{\sqrt{(2 \pi)^{d}|\boldsymbol{R}|}} \mathrm{d} \lambda
\end{aligned}
$$

The first step of the integration consists in isolating the constant terms (i.e. not depending on $\lambda$ ), which leads, if we name $\boldsymbol{\mu}_{b}=\boldsymbol{H} \boldsymbol{\sigma}_{b}$ the errorless set of observations that would be obtained from the first guess source term $\sigma_{b}$, to

$$
\begin{aligned}
& p(\boldsymbol{\mu})=\frac{e^{-\frac{1}{2} \boldsymbol{\mu}^{\mathrm{T}} \boldsymbol{R}^{-1} \boldsymbol{\mu}}}{\theta \sqrt{(2 \pi)^{d}|\boldsymbol{R}|}} \times \\
& \int_{0}^{+\infty} \exp \left(-\frac{1}{2} \boldsymbol{\mu}_{b}^{\mathrm{T}} \boldsymbol{R}^{-1} \boldsymbol{\mu}_{b} \lambda^{2}+\left(\boldsymbol{\mu}^{\mathrm{T}} \boldsymbol{R}^{-1} \boldsymbol{\mu}_{b}-\theta^{-1}\right) \lambda\right) \mathrm{d} \lambda
\end{aligned}
$$

Gaussian integration over $[0,+\infty[$ is given by

$$
\int_{0}^{+\infty} e^{-a x^{2}+b x} \mathrm{~d} x=\sqrt{\frac{\pi}{2}} \frac{e^{\frac{b^{2}}{4 a}}}{\sqrt{2 a}}\left[1-\Phi\left(\frac{-b}{2 \sqrt{a}}\right)\right]
$$

where $\Phi(u)=\frac{2}{\sqrt{\pi}} \int_{0}^{u} e^{-x^{2}} \mathrm{~d} x$ is the error function. In our case, $a=\frac{1}{2} \boldsymbol{\mu}_{b}^{\mathrm{T}} \boldsymbol{R}^{-1} \boldsymbol{\mu}_{b}$ and $b=\boldsymbol{\mu}^{\mathrm{T}} \boldsymbol{R}^{-1} \boldsymbol{\mu}_{b}-\theta^{-1}$, and it leads to Eq. (23), i.e.

$$
\begin{aligned}
p(\boldsymbol{\mu})= & \sqrt{\frac{\pi}{2}} \frac{e^{-\frac{1}{2} \boldsymbol{\mu}^{\mathrm{T}} \boldsymbol{R}^{-1} \boldsymbol{\mu}}}{\theta \sqrt{(2 \pi)^{d}|\boldsymbol{R}|}} \frac{e^{\frac{\left(\mu^{\mathrm{T}} \boldsymbol{R}^{-1} \mu_{b}-\theta^{-1}\right)^{2}}{2 \mu_{b} \boldsymbol{R}^{-1} \mu_{b}}}}{\sqrt{2 \boldsymbol{\mu}_{b}^{\mathrm{T}} \boldsymbol{R}^{-1} \boldsymbol{\mu}_{b}}} \times \\
& {\left[1-\Phi\left(\frac{\theta^{-1}-\boldsymbol{\mu}^{\mathrm{T}} \boldsymbol{R}^{-1} \boldsymbol{\mu}_{b}}{\sqrt{2 \boldsymbol{\mu}_{b}^{\mathrm{T}} \boldsymbol{R}^{-1} \boldsymbol{\mu}_{b}}}\right)\right], }
\end{aligned}
$$

The same calculation is done for $\lambda$ following a Gamma law with $k=2$ (with a further integration by parts) and for $\lambda$ following a semi-Gaussian distribution to obtain respectively Eq. (24) and Eq. (25).

\section{References}

Abida, R., Bocquet, M., 2009. Targeting of observations for accidental atmospheric release monitoring. Atmos. Environ. 43, 6312-6327.

Baklanov, B., Sørensen, J. H., 2001. Parameterisation of radionuclides deposition in atmospheric long-range transport modelling. Phys.Chem.Earth(B) 26 (10), 787-799.

Bocquet, M., 2005a. Reconstruction of an atmospheric tracer source using the principle of maximum entropy. I: Theory. Q. J. Roy. Meteor. Soc. 131, 2191-2208.

Bocquet, M., 2005b. Reconstruction of an atmospheric tracer source using the principle of maximum entropy. II: Applications. Q. J. Roy. Meteor. Soc. 131, 2209-2223.

Bocquet, M., 2007. High resolution reconstruction of a tracer dispersion event. Q. J. Roy. Meteor. Soc. 133, 1013-1026.

Bocquet, M., 2008. Inverse modelling of atmospheric tracers: NonGaussian methods and second-order sensitivity analysis. Nonlin. Processes Geophys. 15, 127-143.

Davoine, X., Bocquet, M., 2007. Inverse modelling-based reconstruction of the Chernobyl source term available for long-range transport. Atmos. Chem. Phys. 7, 1549-1564.

Dee, D., 1995. On-line estimation of error covariance parameters for atmospheric data assimilation. Monthly weather review 123, 1128-1144. 
Delle Monache, L., Lundquist, J. K., Kosovic, B., Johannesson, G., Dyer, K. M., Aines, R. D., Chow, F. K., Belles, R. D., Hanley, W. G., Larsen, S. C., Loosmore, G. A., Nitao, J. J., Sugiyama, G. A., Vogt, P. J., 2008. Bayesian inference and markov chain monte carlo sampling to reconstruct a contaminant source on a continental scale. Journal of Applied Meteorology and Climatology 47, 2600-2613.

Galperin, M. V., 2000. The approaches to correct computation of airborne pollution advection. In: Problems of Ecological Monitoring and Ecosystem Modelling. XVII, St. Petersburg, Gidrometeoizdat, 2000. pp. 54-68.

Genikhovich, E., Sofiev, M., Gracheva, I., 2004. Interactions of meteorological and dispersion models at different scales. Springer, pp. $158-166$.

Issartel, J.-P., 2003. Rebuilding sources of linear tracers after atmospheric concentration measurements. Atmos. Chem. Phys. 3, 2111-2125.

Issartel, J.-P., Baverel, J., 2003. Inverse transport for the verification of the comprehensive nuclear test ban treaty. Atmos. Chem. Phys. 3, 475-486.

Krysta, M., Bocquet, M., 2007. Source reconstruction of an accidental radionuclide release at European scale. Q. J. Roy. Meteor. Soc. 133, 529-544.

Krysta, M., Bocquet, M., Brandt, J., 2008. Probing ETEX-II data set with inverse modelling. Atmos. Chem. Phys. 8, 3963-3971.

Liu, D., Nocedal, J., 1989. On the limited memory method for large scale optimization. Math. Program. B. 45(3), 503-528.

Louis, J., 1979. A parametric model of vertical eddy fluxes in the atmosphere. Boundary-Layer Meteor. 17, 197-202.

Nodop, K., Connolly, R., Girardi, F., 1998. The field campaigns of the European Tracer Experiment (ETEX): Overview and results. Atmos. Environ. 32, 4095-4108.

Politis, K., Robertson, L., 2004. Bayesian updating of atmospheric dispersion after a nuclear accident. Appl. Statist. 53, 583-600.

Quélo, D., Krysta, M., Bocquet, M., Isnard, O., Minier, Y., Sportisse, B., 2007. Validation of the Polyphemus platform on the ETEX, Chernobyl and Algeciras cases. Atmos. Env. 41, 5300-5315.

Robertson, L., Langner, J., 1997. Variational assimilation of ETEX-I data. In: Nodop, K. (Ed.), Proceedings of the ETEX Symposium on long-range atmospheric transport, model verification and emergency response.

Saunier, O., Bocquet, M., Matthieu, A., Isnard, O., 2009. Model reduction via principal component truncation for the optimal design of atmospheric monitoring networks. Atmos. Env. 43, 4940-4950.

Seibert, P., 2001. Inverse modelling with a lagrangian particle dispersion model: application to point releases over limited time intervals. In: Air Pollution Modeling and its Application XIV. Plenum Press.

Sofiev, M., 2000. A model for the evaluation of long-term airborne pollution transport at regional and continental scales. Atmos. Env. 34, 2481-2493.

Sofiev, M., 2002. Extended resistance analogy for construction of the vertical diffusion scheme for dispersion models. J. Geophys. Res. 107(D12), 4159

Sofiev, M., Siljamo, P., Valkama, I., Ilvonen, M., Kukkonen, J., 2006. A dispersion modelling system silam and its evaluation against etex data. Atmos. Env. 40, 674-685.

Wesely, M. L., 1989. Parametrization of surface resistances to gaseous dry deposition in regional-scale numerical models. Atmos. Env. 23, 1293-1304.

Yee, E., Lien, F.-S., Keats, A., D’Amours, R., 2008. Bayesian inversion of concentration data: Source reconstruction in the adjoint representation of atmospheric diffusion. Journal of Winf Engineering and Industrial Aerodynamics 96, 1805-1816.

Zhang, L., Gao, S., Padro, J., Barrie, L., 2001. A size-segregated dry deposition scheme for an atmospheric aerosol module. Atmos. Env. $35,549-560$. 

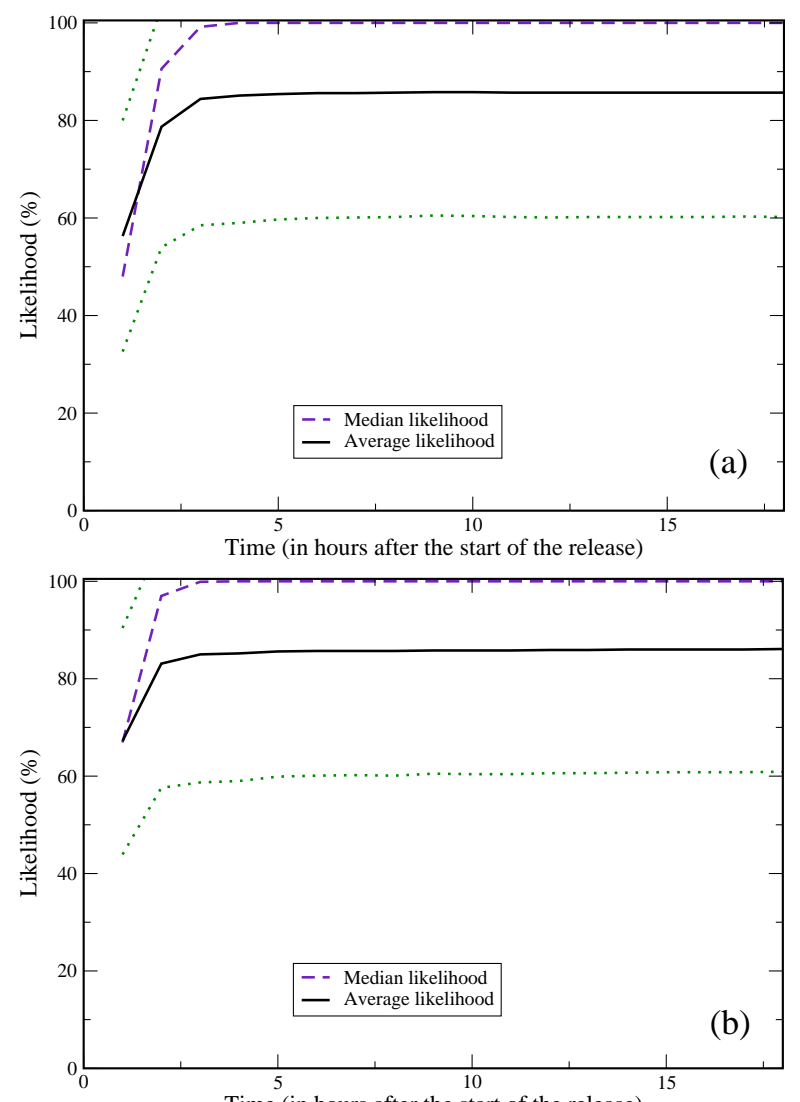

Time (in hours after the start of the release)

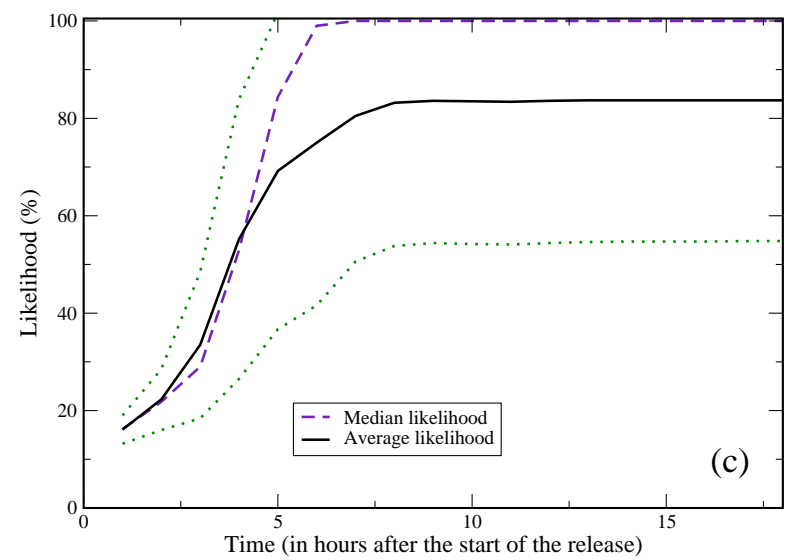

Figure 8: Mean normalised trace of the likelihood matrix $\boldsymbol{L}$ (full line) as compared to the median normalised trace (dashed line). The upper and lower curves indicate the standard deviation range of the normalised trace. Panel (a): in the case of the Gaussian Bayesian test. Panel (b): in the semi-Gaussian case of the non-Gaussian Bayesian test, with the prior $\sigma_{b}$ equal to the true source term. Panel (c): in the semi-Gaussian case of the non-Gaussian Bayesian test, with the prior $\sigma_{b}$ taken as a constant term, equal to the mean of the true source. 


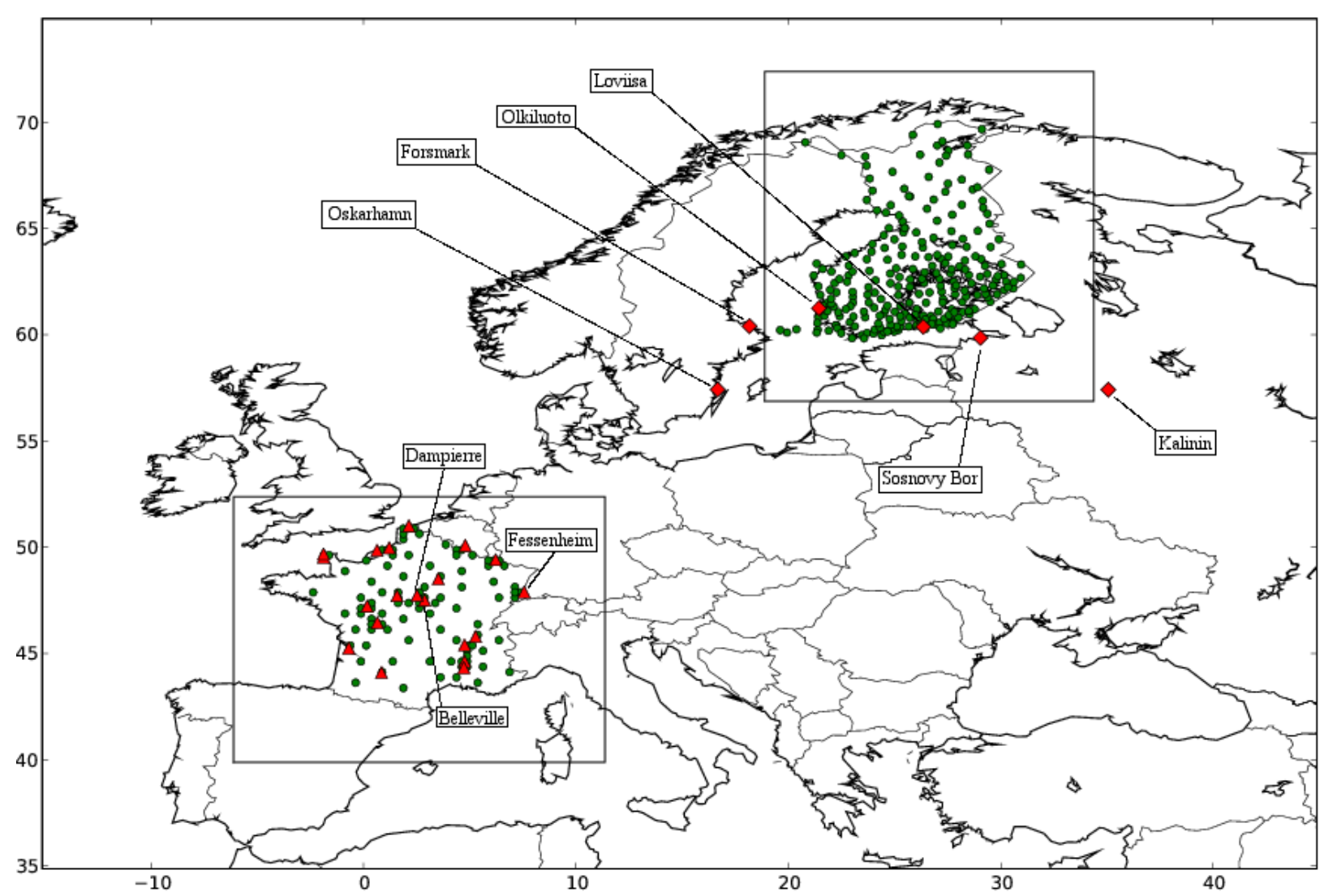

Figure 1: Representation of the domain of study and of the two subdomains centred on France and Finland. The black dots represent the monitoring stations: the OPERA-AIR network which is virtual in the French case (optimal network to be built), or the Finnish monitoring network. The locations of the civil nuclear sites are indicated by triangles and rhombuses respectively. 

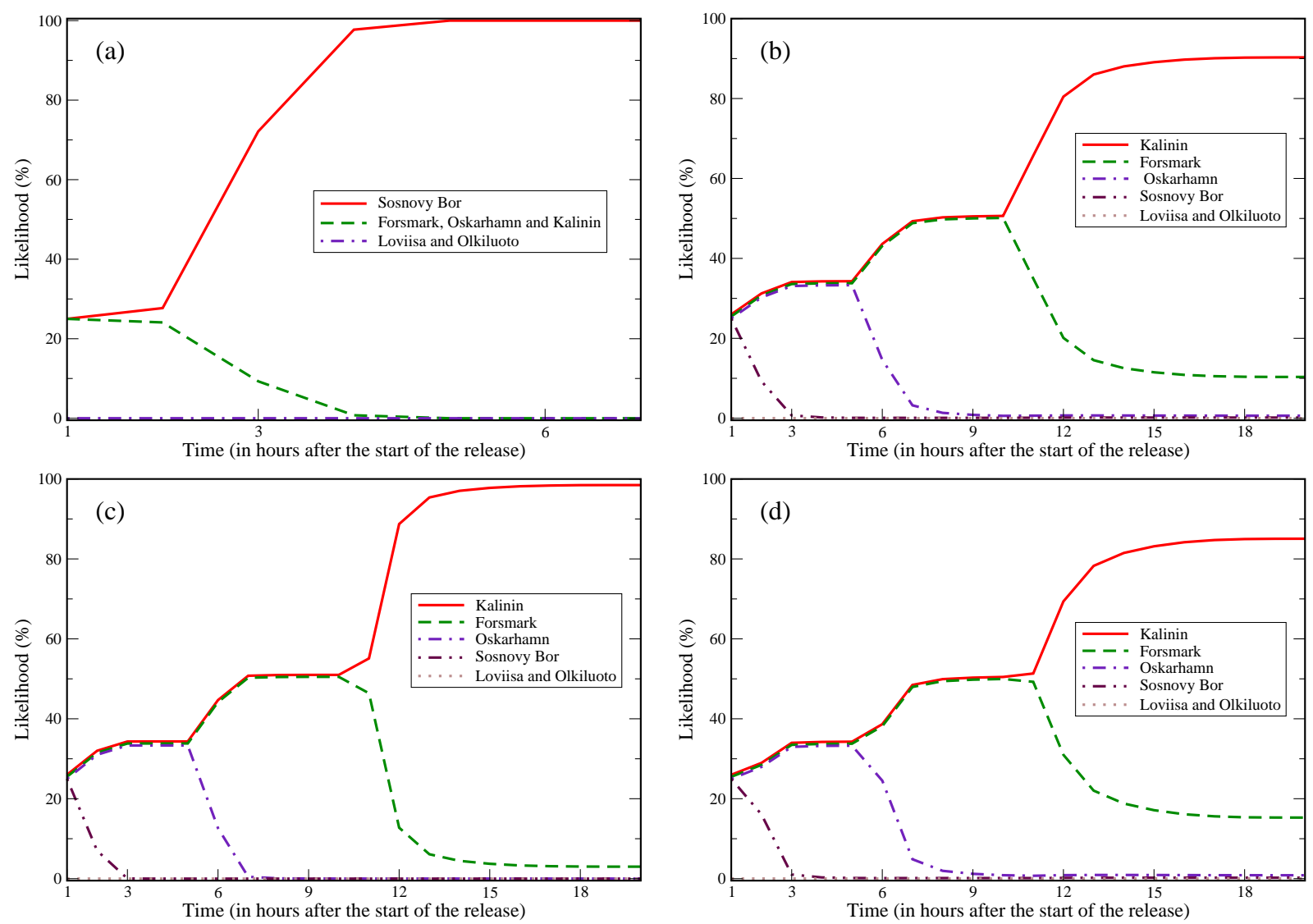

Figure 6: Panel (a): Gaussian Bayesian tests on the Sosnovy Bor case. Panel (b), (c), and (d): non-Gaussian Bayesian tests on the Kalinin case. Panel (b) corresponds to the exponential, $k=1$ case. Panel (c) corresponds to the exponential, $k=2$ case. Panel (d) corresponds to the semi-Gaussian case. 